\title{
Microchromosomes are building blocks of bird, reptile, and mammal chromosomes
}

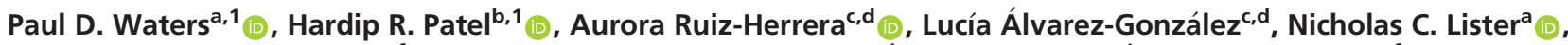

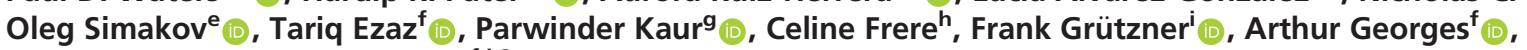 \\ and Jennifer A. Marshall Graves ${ }^{\mathrm{f}, \mathrm{j}, 2}$ (i)
}

aSchool of Biotechnology and Biomolecular Science, Faculty of Science, UNSW Sydney, Sydney, NSW 2052, Australia; ${ }^{\text {b} T h e ~ J o h n ~ C u r t i n ~ S c h o o l ~ o f ~ M e d i c a l ~}$ Research, Australian National University, Canberra, ACT 2601, Australia; ${ }^{C}$ Departament de Biologia Cel.lular, Fisiologia i Immunologia, Universitat Autònoma de Barcelona, Cerdanyola del Vallès 08193, Spain; ${ }^{\mathrm{d}}$ Genome Integrity and Instability Group, Institut de Biotecnologia i Biomedicina, Universitat Autònoma de Barcelona, Cerdanyola del Vallès 08193, Spain; ' ${ }^{\circ}$ epartment of Neurosciences and Developmental Biology, University of Vienna, 1010 Vienna, Austria; IInstitute for Applied Ecology, University of Canberra, Canberra, ACT 2601, Australia; ${ }^{9}$ UWA School of Agriculture and Environment, The University of Western Australia, Crawley, WA 6009, Australia; h Global Change Ecology Research Group, University of the Sunshine Coast, Sippy Downs, QLD 4556, Australia; ischool of Biological Sciences, University of Adelaide, Adelaide, SA 5000, Australia; and 'School of Life Sciences, La Trobe University, Bundoora, VIC 3068, Australia

This contribution is part of the special series of Inaugural Articles by members of the National Academy of Sciences elected in 2019.

Contributed by Jennifer A. Marshall Graves, August 27, 2021 (sent for review July 9, 2021; reviewed by Darren Griffin and Josquin F. G. Tibbits)

Microchromosomes, once considered unimportant shreds of the chicken genome, are gene-rich elements with a high GC content and few transposable elements. Their origin has been debated for decades. We used cytological and whole-genome sequence comparisons, and chromosome conformation capture, to trace their origin and fate in genomes of reptiles, birds, and mammals. We find that microchromosomes as well as macrochromosomes are highly conserved across birds and share synteny with single small chromosomes of the chordate amphioxus, attesting to their origin as elements of an ancient animal genome. Turtles and squamates (snakes and lizards) share different subsets of ancestral microchromosomes, having independently lost microchromosomes by fusion with other microchromosomes or macrochromosomes. Patterns of fusions were quite different in different lineages. Cytological observations show that microchromosomes in all lineages are spatially separated into a central compartment at interphase and during mitosis and meiosis. This reflects higher interaction between microchromosomes than with macrochromosomes, as observed by chromosome conformation capture, and suggests some functional coherence. In highly rearranged genomes fused microchromosomes retain most ancestral characteristics, but these may erode over evolutionary time; surprisingly, de novo microchromosomes have rapidly adopted high interaction. Some chromosomes of early-branching monotreme mammals align to several bird microchromosomes, suggesting multiple microchromosome fusions in a mammalian ancestor. Subsequently, multiple rearrangements fueled the extraordinary karyotypic diversity of therian mammals. Thus, microchromosomes, far from being aberrant genetic elements, represent fundamental building blocks of amniote chromosomes, and it is mammals, rather than reptiles and birds, that are atypical.

vertebrate chromosome evolution | whole-genome alignment chromosome conformation | microchromosome origin | amphioxus

C lassic cytological studies described mammalian chromosomes of a size easily visible under the microscope. Bird and reptile karyotypes were strikingly different, with a size discontinuity between macrochromosomes, with sizes ( 3 to $6 \mu \mathrm{m})$ in the range of mammalian chromosomes and microchromosomes $(<0.5 \mu \mathrm{m})$ which looked more like specks of dust (e.g., refs. 1-3). These microchromosomes stained oddly and occupied a central position at mitosis (4).

An early view of microchromosomes as inconstant heterochromatic elements (5), or even not chromosomes at all, was thoroughly debunked (1, 6-8). Like macrochromosomes, they possess a centromere and telomeres at each end (with extralong subtelomeric repeats) (9) and segregate regularly at mitosis.
Microchromosomes are GC-rich and gene-dense with a low content of repetitive sequence (10) and have high rates of recombination. They replicate early and are hyperacetylated compared to macrochromosomes, suggesting they are highly transcribed.

At the cytological level, most birds have extremely conserved karyotypes, including 9 pairs of macrochromosomes and 30 to 32 pairs of microchromosomes (3), defined by relative sizes where there is no abrupt size discontinuity. Chromosome constitutions of birds are listed in ref. 11. Although there are some spectacular exceptions, especially in the highly rearranged falcon and the parrot genomes, even distantly related birds such as chicken and emu share nine macrochromosome pairs

\section{Significance}

Genomes of birds and reptiles, but not mammals, consist of a few large chromosomes and many tiny microchromosomes. Microchromosomes are gene-rich and highly conserved among birds and reptiles and share homology with one or more of the tiny chromosomes of an invertebrate that diverged from the vertebrate lineage $684 \mathrm{Ma}$. Microchromosomes interact strongly and crowd together at the center of cells, suggesting functional coherence. Many microchromosomes have been lost independently in turtles, snakes, and lizards as they have fused with each other or with larger chromosomes. In mammals they have completely disappeared, yet some chromosomes of the basal platypus line up with several microchromosomes, suggesting that they are the building blocks of the atypically variable chromosomes of mammals.

Author contributions: J.A.M.G. designed research; P.D.W., H.R.P., A.R.-H., L.A.-G., T.E., C.F., F.G., and A.G. performed research; H.R.P., A.R.-H., P.K., C.F., and F.G. contributed new reagents/analytic tools; P.D.W., H.R.P., A.R.-H., N.C.L., O.S., T.E., A.G., and J.A.M.G. analyzed data; P.D.W., H.R.P., and J.A.M.G. wrote the paper; and P.K. performed genome assembly of DNA Zoo species using high-throughput chromosome conformation capture.

Reviewers: D.G., University of Kent; and J.F.G.T., Agriculture Victoria.

Competing interest statement: Four authors (J.A.M.G., A.R.-H., T.E., and F.G.), as well as one of the referees, made contributions to a review of the continued usefulness of cytogenetics in the age of genomics, solicited and edited by Janine Deakin (https:// www.mdpi.com/2073-4425/10/8/627).

This open access article is distributed under Creative Commons Attribution License 4.0 (CC BY).

See Profile, e2116850118, in vol. 118, issue 43.

${ }^{1}$ P.D.W. and H.R.P. contributed equally to this work.

${ }^{2}$ To whom correspondence may be addressed. Email: j.graves@latrobe.edu.au.

This article contains supporting information online at https://www.pnas.org/lookup/ suppl/doi:10.1073/pnas.2112494118/-/DCSupplemental.

Published November 1, 2021 
identified by banding patterns, chromosome painting, and gene mapping $(8,12-15)$.

Microchromosomes are too small to distinguish morphologically, let alone by G-band patterns, and pairing them is mostly guesswork. However, their number is usually constant and even, as expected for paired autosomes in diploids. Cytological examination, using specific DNA probes, suggests conservation of microchromosomes across 22 avian species (16), and comparative gene mapping and whole-genome analysis attests to considerable conservation among distantly related bird groups $(17,18)$. A few bird species have more microchromosomes, but chromosome painting reveals their recent origin from fission (14). Genome sequencing of many bird species now provides unprecedented detail sufficient to compare microchromosomes across avian species (19).

Fewer comparative studies of microchromosome conservation have been done in reptiles, but their genome structures are similar to those of birds, many with an abrupt distinction between a few macrochromosomes and many microchromosomes (reviewed in refs. 20-22). However, turtles and snakes have fewer microchromosomes than birds. There is G-band and chromosome painting homology between the macrochromosomes of birds and turtles (23), and a close relationship between the chromosomes of birds and squamates (snakes and lizards) was noted early (24). Gene mapping and sequence comparisons reveal many homologous synteny blocks $(8,25)$, and sequence comparisons show that several microchromosomes are conserved at the sequence level (26). Lizard karyotypes are more variable; some species have clearly demarcated macro- and microchromosomes, whereas others show no clear distinction.

There are exceptional reptile and bird clades in which no abrupt size difference defines microchromosomes, and the size range of microchromosomes can also vary between clades. For example, eagle and parrot genomes have few microchromosomes (27) and crocodilians have five very large macrochromosomes and few chromosomes in the microchromosome size range (28).

The origin of microchromosomes has been debated for decades. Initially they were thought to represent some sort of breakdown product of "normal" mammalian-like macrochromosomes that existed in amniote, even tetrapod, ancestors (29), and this view is still expressed (e.g., ref. 30). The alternative view is that at least some of them represent the small chromosomes of a vertebrate ancestor $400 \mathrm{Ma}$, retained intact by several vertebrate clades $(8,26,31)$. Similarities with the small chromosomes of amphioxus (the lancelet, an early branching chordate) now suggest a much earlier origin (32), dating back to at least $684 \mathrm{My}$ since they last shared a common ancestor with vertebrates.

With the availability of several chromosome-scale assemblies of bird and reptile genomes $(10,19)$, it is now possible to trace the origin and fate of microchromosomes in birds, reptiles, and mammals. We compared the genomes of 7 birds and 10 reptiles with chromosome-level assemblies, as well as three mammals and an amphioxus (Fig. 1). These comparisons provide evidence that, indeed, microchromosomes represent a set of highly conserved ancient animal chromosomes, whereas macrochromosomes, which are considered "normal" because of their ubiquity in mammals, have undergone multiple lineage-specific rearrangements, especially in mammals. We gather evidence that microchromosomes retain a high frequency of interchromosome interaction inside the nucleus and regularly locate together at interphase and division, suggesting retention of an ancestral functional coherence between a set of small ancestral chromosomes.

\section{Results}

Cytological Observations of Microchromosomes. To broaden our knowledge of microchromosome cytology we examined the microchromosomes of several reptiles, including snakes (python and tigersnake), lizards (spiny tailed monitor and our model, the central bearded dragon Pogona vitticeps), and a turtle (eastern long-neck). We found that microchromosomes were less strongly stained and tended to clump centrally in mitotic and meiotic cells (e.g., in bearded dragon and turtle; Fig. $2 A$ and $B$ ). Several repetitive sequences specifically hybridize to most or all the microchromosomes, allowing us to detect their central position also within the interphase nucleus (Fig. 2C).

We also used DNA paints that specifically hybridized to two bearded dragon microchromosome pairs (Fig. 2D) to explore their conservation in other species. We found that in the eastern long-neck turtle the two paints hybridized together at the tip of a macrochromosome, implying that these two sequences are fused, and fused to a macrochromosome in the turtle lineage; significantly, the paints hybridized to central regions of interphase nuclei in turtle as well as bearded dragon (Fig. 2E).

Together our observations support the view that microchromosomes are differentiated from macrochromosomes, not only by their much smaller size but also by their different staining properties (denoting different sequence makeup and chromosome conformation) and their location together in the center of the interphase nucleus and dividing cells.

Genome Sequence Comparisons. We performed pairwise wholegenome alignments using LastZ (33) to identify syntenic blocks (reciprocally best aligned chains) and show conservation of chromosomes from birds, turtles, and squamates within and between lineages. The relationships of the species we used are presented in Fig. 1. We define microchromosome according to published karyotypes (see detailed information about species and their chromosomes in SI Appendix, Table S1). In birds and snakes all assembled macrochromosomes were smaller than 35 $\mathrm{Mb}$. In other clades the threshold size of the largest microchromsome was greater: turtles $(45 \mathrm{Mb})$, lizards with rearranged genomes $(50 \mathrm{Mb})$, tegu $(75 \mathrm{Mb})$, and alligator $(96 \mathrm{Mb})$. Sequence comparisons of bird and turtle genomes. Of the bird species with chromosome-scale assembled genomes, we chose emu, chicken, pigeon, jackdaw, and hummingbird, species with conserved karyotypes that captured the deepest avian divergences (Fig. 1). Genomes of these five birds display striking homology, aligning over $>87 \%$ of their length (Fig. $3 A$ ). The nine macrochromosomes (eight autosomes and the $\mathrm{Z}$ sex chromosome) are almost invariant. We observed no fusions of macrochromosomes and only two macrochromosomes in humming bird and one in jackdaw that have undergone fission. We conclude that macrochromosomes are highly conserved in birds.

There was little variation (28-31) in the number of microchromosomes across these five birds (Fig. 1 and SI Appendix, Tables S1 and S2). Sequence comparisons of assembled microchromosomes show that they too are highly conserved, nearly all showing a 1:1 correspondence between all five bird species (Fig. $3 A$ ). The most prominent exception is a microchromosome in all other bird lineages that aligns to chicken chromosome $4 p$, as previously noted $(8,12)$, and is significant because it also has homology to the conserved region of the mammalian $\mathrm{X}$ chromosome (34). Uniquely, the hummingbird genome contains two chromosomes in the microchromosome size range that are parts of macrochromosomes in other birds, implying an origin by fission of macrochromosomes. Conserved microchromosomes are GC-rich and gene-dense in all species (mean of 40 genes per $\mathrm{Mb}$ compared to 17 genes per $\mathrm{Mb}$ on macrochromosomes in chicken) (Fig. 4 and SI Appendix, Fig. S1).

We conclude that microchromosomes and macrochromosomes are highly conserved between even the most distantly related bird lineages separated by $\sim 110 \mathrm{My}$. 


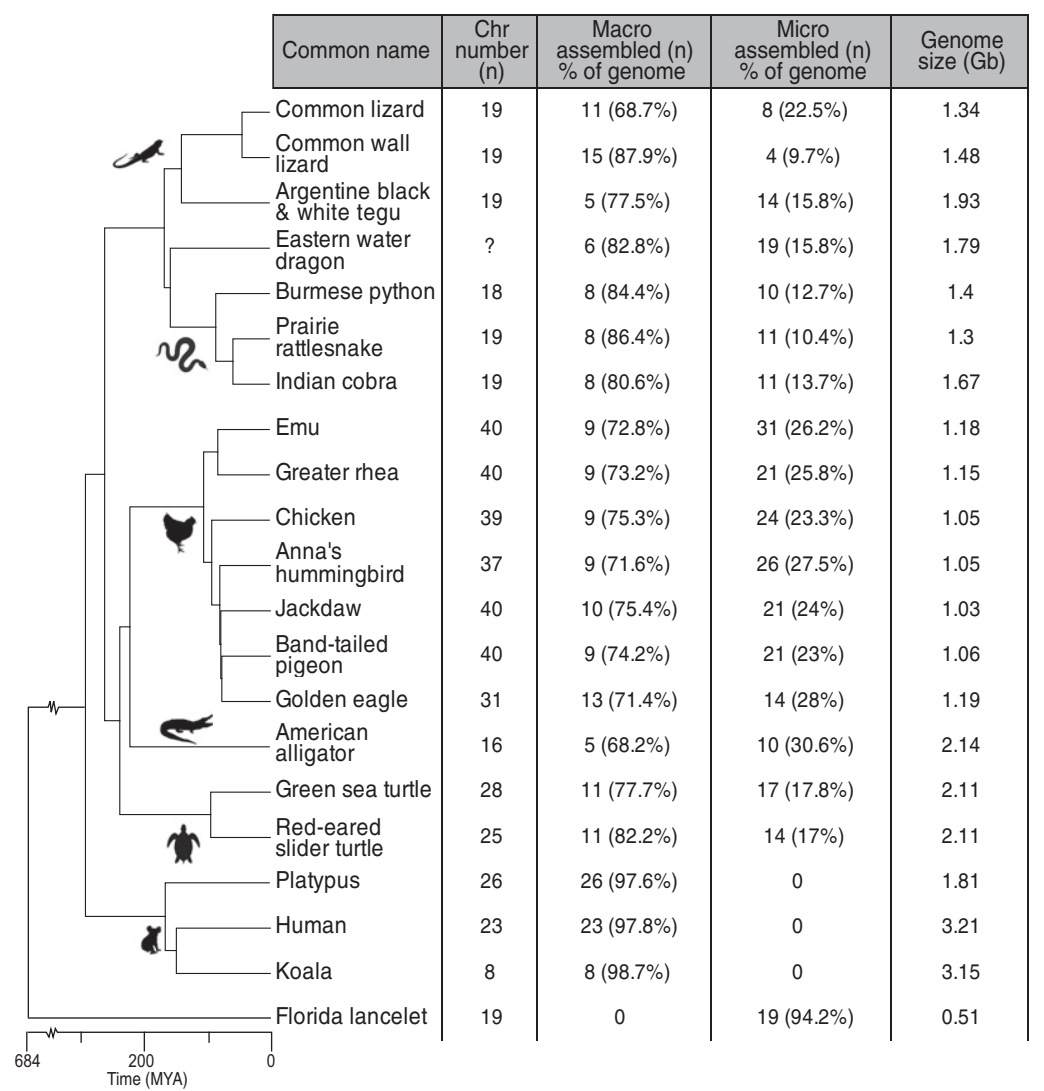

Fig. 1. Phylogenetic relationships of reptiles, birds, mammals, and amphioxus genome assemblies compared in this study. Cytological chromosome numbers ( $n$ ) are shown, along with the number of assembled macrochromosomes and microchromosomes (their percentage of the anchored genome) and genome size. Species names and full common names are given in SI Appendix, Table S1; in the text they are referred to by abbreviated common names.

We then broadened our comparison to include turtle genomes. Chromosome-level assemblies of two turtle species were available, the green sea turtle and the red-eared slider turtle, with almost identical karyotypes. As in birds, turtle microchromosomes have a higher GC content than macrochromosomes (SI Appendix, Fig. $\mathrm{S} 1 A$ ). Sequence comparison shows 1:1 correspondence between two turtle genomes (Fig. $3 A$ ) except for two green sea turtle microchromosomes that align to parts of large macrochromosomes in the slider.

Turtle macrochromosomes align to emu macrochromosomes with some intrachromosomal rearrangements. However, turtles have seven fewer microchromosomes. There is an apparent fusion of two microchromosomes conserved between sea turtle and emu that results in a larger microchromosome in the slider turtle. Four emu microchromosomes are present as two macrochromosomes in both turtle species. Two emu microchromosomes are fused to the termini of different macrochromosomes in sea turtle and slider, and one is fused to a macrochromosome in the sea turtle only.

Thus, genomes in turtles, as well as birds, are highly conserved. Hereafter we use the emu genome (35) as a representative of the ancestral bird state and the green sea turtle to represent the turtle ancestral state, for interclade comparisons.

Sequence comparisons of squamate genomes. Several genomes of snakes (python, rattlesnake, and cobra) and lizards (common lizard, wall lizard, the distantly related tegu, and the outgroup water dragon) have been recently sequenced and have chromosome-scale assembled microchromosomes to enable detailed comparisons and definition of ancestral states. The size differences between macro- and microchromosomes were obvious in the snakes, water dragon, and tegu but less pronounced in the common and wall lizards. However, GC content and gene density of microchromosomes was higher than for the macrochromosomes in all these species (SI Appendix, Fig. S1).

Sequence comparisons of snake genomes (Fig. $3 B$ ) showed that macro- and microchromosomes are almost wholly conserved between python, rattlesnake, and cobra. There were relatively few macrochromosome fusion/fission events between these species. The 10 microchromosomes, too, were all conserved between the three snake species, apart from some minor rearrangements. However, notably, a cobra-specific microchromosome aligns to part of a small macrochromosome in other snake species.

The water dragon, which is more closely related to snakes than the other lizards studied (36), shares the snake macroand microchromosome structure except for two microchromosomes that align to termini of the largest snake chromosome. Four water dragon microchromosomes had no alignments to any of the snake or lizard genomes. Chromosomes of the water dragon and tegu were almost identical (Fig. $3 B$ ).

The common lizard and the wall lizard both had more and smaller macrochromosomes than tegu, water dragon, or snakes; 10 of these align to the five largest tegu and water dragon macrochromosomes, suggesting they are the products of fission. Both had few chromosomes in the microchromosome size range, and their alignments to multiple microchromosomes conserved between snakes, water dragon, and tegu suggest fusion of two or three microchromosomes. There was also evidence of a triply fused microchromosome fused with another microchromosome in the wall lizard to form a chromosome out of the microchromosome range.

There is evidence, too, of microchromosomes arising de novo from chromosome fission. Four novel microchromosomes in the wall lizard have homology to two macrochromosomes in the common lizard, which have homology to parts of two larger 


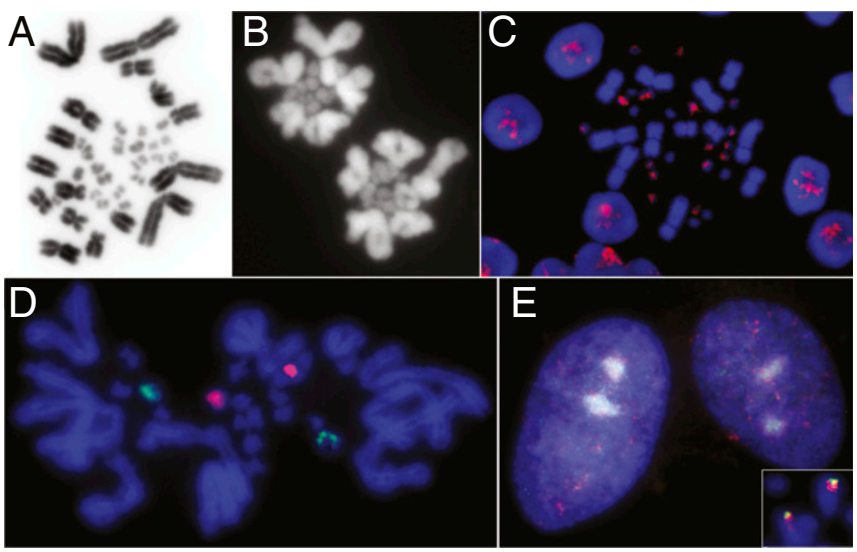

Fig. 2. Cytological characterization of microchromosomes in reptiles. $(A)$ Mitotic chromosomes of the bearded dragon, showing extreme size difference, different staining, and central location of microchromosomes. Image credit: Jason Dobry (La Trobe University, Melbourne, Australia). (B) Polar view of diakinesis in bearded dragon spermatocyte, showing extreme size and staining difference and central location of macro and micro bivalents. (C) Fluorescence in situ hybridization using microchromosome-specific repetitive sequence $[A G A T]_{n}$ showing central clustering of labeled dragon microchromosomes in interphase nuclei. (D) Two probes (green and red) light up two pairs of microchromosomes in dragon cells. $(E)$ The same probes retain their central location in interphase nuclei, although they colocate to the terminus of a macrochromosome in the long-neck turtle (Inset).

macrochromosomes in the tegu. Another microchromosome shared by both lizards has homology to another region of the same tegu macrochromosome.

Thus, microchromosomes are very conserved among snakes, water dragon, and tegu and must represent the ancestral state. However, the wall lizard and common lizard lineages have undergone fusions (largely micromicro) and a few fissions. We use the python and tegu to represent the ancestral state for lizards and snakes in across-clade comparisons.

Sequence comparisons between birds and reptiles suggest that bird microchromosomes best represent the ancestral amniote condition. We used pairwise genome alignments of the representative bird, turtle, snake, and lizard for across-clade comparisons (Fig. 3C and SI Appendix, Fig. S2). There was striking homology between the macrochromosomes and also between the microchromosomes of emu, green turtle, python, and tegu.

The genome sizes of turtles, snakes, and lizards were larger than those of birds (Fig. 1), suggesting that the sequence was expanded by the insertion and retention of repetitive elements. Comparisons of scaled genomes (Fig. 3C) suggest that this expansion affected macrochromosomes and microchromosomes equally. Notably, a few emu microchromosomes expanded into the range of macrochromosomes in turtles or squamates; for instance, one microchromosome in tegu has 1:1 homology to a small chromosome in the common and wall lizards, but because it is larger than $50 \mathrm{Mb}$ (our threshold for microchromosome size in squamates; see SI Appendix, Table S1) it is classified as a macrochromosome in the wall (but not the common) lizard (Fig. 3C).

Turtles have fewer microchromosomes than birds. Of the 22 assembled emu microchromosomes, only 15 are conserved as microchromosomes in the green turtle. A microchromosome present in the two turtle assemblies is missing from the emu and chicken assemblies. Four emu microchromosomes are present as micromicro fusions. The other two emu microchromosomes align to arms of macrochromosomes in turtles, suggesting macromicro fusion. Thus, turtles retain a subset of microchromosomes homologous to those of emu. Others are fused either with another microchromosome to form larger (micro- or macrochromosome-scale) entities or are fused to bird macrochromosomes.

Our representative squamates also have fewer microchromosomes than birds. Python has 10 and tegu 12 (plus 2 that align to a single macrochromosome in python and birds). The 10 snake microchromosomes (and their tegu counterparts) all align to bird microchromosomes. Other bird microchromosomes align to autosomes in python and tegu, one to a terminal position and seven others make up two chromosome arms. Thus squamates, too, share a subset of bird microchromosomes.

Importantly, although nearly all of the microchromosomes in turtle and squamate assemblies are homologous to bird microchromosomes they represent different subsets. Of $21 \mathrm{emu}$ microchromosomes with sufficient homology to regions of both tegu and turtle genomes, only eight are microchromosomes in both (Fig. 3C). Three are incorporated into different macrochromosomes in tegu and turtle. Seven emu microchromosomes have homology to turtle microchromosomes but squamate macrochromosomes, and three emu microchromosomes have homology to squamate microchromosomes but turtle macrochromosomes.

The simplest explanation of the pattern of microchromosome retention is that birds represent the ancestral amniote condition (31 microchromosome pairs) and micromicro and micromacro fusions reduced the numbers of microchromosomes independently in turtles and squamates.

Inspection of the bird microchromosomes with homology to macrochromosomes in turtles or squamates (or both) reveal different patterns of microchromosome fusion and fission. Among the bird microchromosomes with homology to regions of turtle macrochromosomes, there are fusions of two or three microchromosomes that generate larger (macrochromosomesized) chromosomes. For instance, the two smallest turtle macrochromosomes are each homologous to two bird microchromosomes, implying fusions of ancestral microchromosomes. Other microchromosomes, or micromicro fusions, have fused to terminal regions of macrochromosomes in turtles or squamates. Several appear to be Robertsonian (centric) fusions, in which an ancestral microchromosome or micromicro fusion has become a macrochromosome arm (e.g., the turtle macrochromosome pair pictured in Fig. 2E). Almost all other fusions are terminal, with very few examples of internal integration into a macrochromosome.

While the predominant evolutionary pattern is of continued loss of microchromosomes by fusion, a few novel microchromosomes have been created by fission from macrochromosomes, for example independently in hummingbird, cobra, and common lizard.

To ensure that our inference about microchromosome conservation is not an artifact of selected pairwise comparisons, we calculated the percentage of microchromosome aligned regions in each query species, which aligned to microchromosomes of another target species (Fig. 3D). We observed that 72 to $100 \%$ of microchromosome alignments for all species (except wall lizard and golden eagle) are to bird microchromosomes. When chicken (fusion of a microchromosome to chromosome 4) and hummingbird (fission of macrochromosomes that result in new microchromosomes) are excluded, $>98 \%$ are microto-micro alignments between birds. It is intriguing to note that the heat map (Fig. 3D) is not symmetrical. This is because when birds are the target genome most microchromosomes of other species align to bird microchromosomes. However, many of their microchromosomes have been fused into macrochromosomes in other clades, so when birds are the query genome the microto-micro alignment proportion is reduced. This demonstrates that the plot order of species in Fig. 3 has no influence 
A

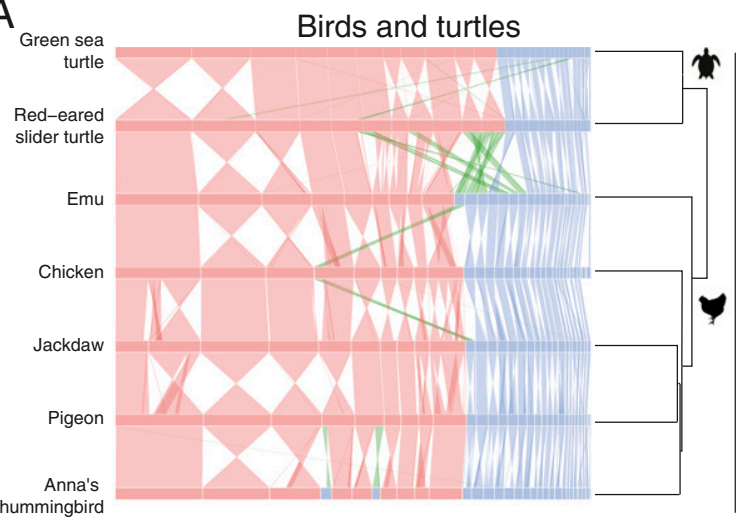

B

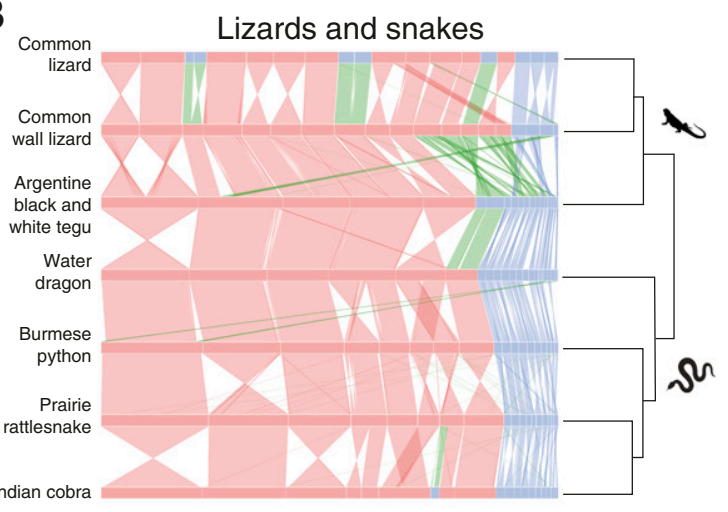

C

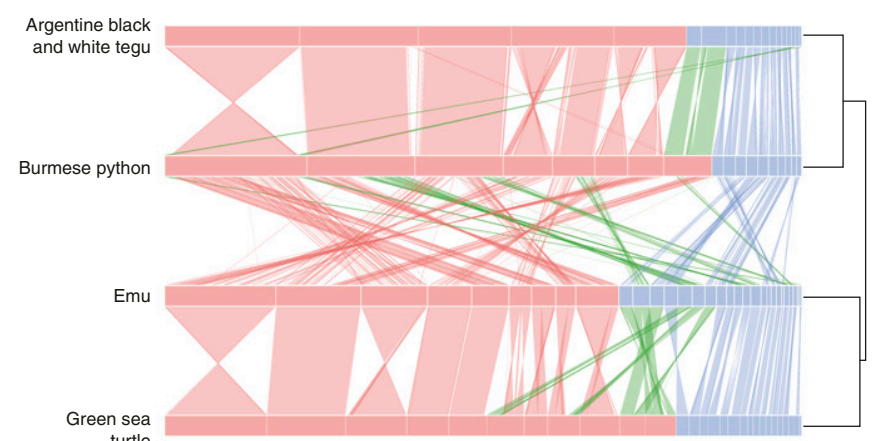

D Microchromosome conservation

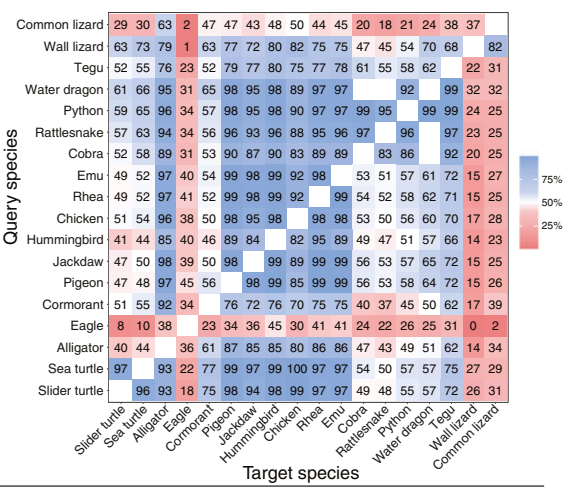

Target species
E

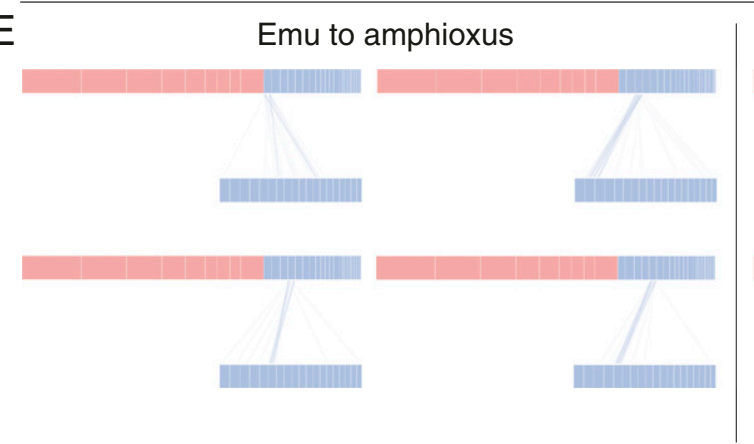

\section{$\mathrm{F} \quad$ Amphioxus to emu}
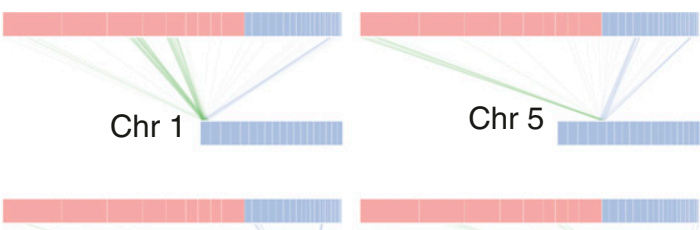

Chr 14

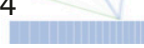

Chr 16

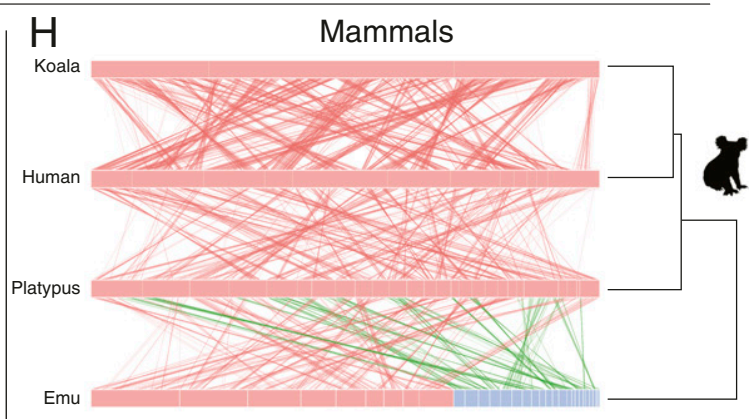

alligator

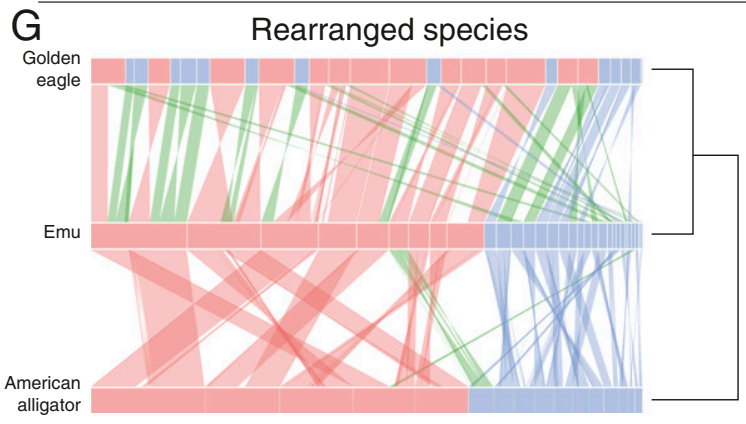

Rearranged species

Macro to macro homology

Macro to micro homology

Micro to micro homology

Fig. 3. Sequence homology plots within and between birds, reptiles, and mammals and comparison to the chordate amphioxus. Relationships between species and genome sizes are shown on the right. Genomes are resized, with chromosome sizes depicted as a proportion of genome length. Some chromosomes have been reordered in the plots to better show homologies within and between clades (see SI Appendix, Table S2). Macrochromosomes are shown in red and microchromosomes in blue; changes between these states are shown in green. Chained and netted alignments were filtered for a minimum length of $100 \mathrm{~kb}$ for vertebrate species and $5 \mathrm{~kb}$ for amphioxus. Sequence homology between macro- and microchromosomes within $(A)$ birds and turtles and $(B)$ squamates. (C) Sequence homology between macro and microchromosomes of a representative lizard (tegu), snake (python), bird (emu), and the green sea turtle. $(D)$ Heat map showing the fraction (as a function of alignment chain length) of microchromosomes in the query species ( $y$ axis) that align to microchromosomes in the target species ( $x$ axis). ( $E$ and $F$ ) Comparisons of emu and amphioxus chromosomes: Single emu microchromosomes have homology to one (or two) amphioxus chromosomes (E), single amphioxus chromosomes detect strong homology to one (or more) emu microchromosomes (from left to right DNAzoo scaffolds 9, 10, 29 and 8), as well as to macrochromosome regions (F). (G) Sequence comparisons between emu and the rearranged alligator and eagle genomes. $(H)$ Sequence comparisons between emu and mammals: eutherian (human), marsupial (koala), and monotreme (platypus) 

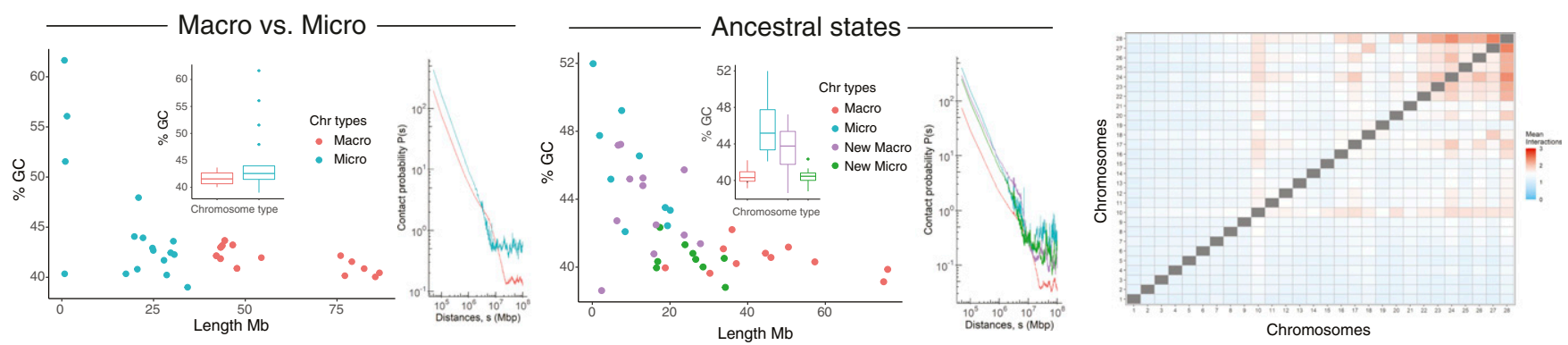

Fig. 4. GC content versus size and distance-dependent interaction probabilities (Ps) of golden eagle macro- and microchromosomes in the present derived state, and after partitioning the genome into ancestral micro- and macrochromosomes and new micro and macrochromosomes. The interchromosome interaction (with scaffolds sorted from largest to smallest, blue is low interaction and red high) heat map is for the present derived state.

on our interpretation of microchromosome conservation and ancestral state.

Origin of Microchromosomes: Homology with Amphioxus Chromosomes. If we accept that the division of the bird genome into macro- and microchromosomes represents the ancestral amniote condition, the occurrence of microchromosomes in some fish $(32,37)$ suggests this characteristic might be ancestral to all vertebrates. Indeed, ancestral vertebrates may have possessed even more microchromosomes which fused before the divergence of the sauria.

However, did microchromosomes arise by fission of larger chromosomes in an ancient chordate ancestor, or did larger vertebrate chromosomes arise by fusion of ancestral microchromosomes? Or did both processes occur (8)? To address this question, we compared conserved bird and reptile microchromosomes with the small chromosomes of a distant chordate relative, the amphioxus (Florida lancelet, Branchiostoma floridae), which last shared a common ancestor with vertebrates $684 \mathrm{Ma}$.

Amphioxus has a small $(520 \mathrm{Mb})$ genome divided into 19 tiny chromosomes that range in size from $17 \mathrm{Mb}$ to $35 \mathrm{Mb}$. These chromosomes are very gene-dense (60 genes per $\mathrm{Mb}$ compared to 10 per Mb in mammals; SI Appendix, Fig. S1H). Comparison of the amphioxus sequence with those of garfish and chicken revealed two genome doublings: an autotetraploidization in the Cambrian $\sim 500 \mathrm{Ma}$ and allotetraploidy by fusion of genomes that had diverged in a fish ancestor 460 $\mathrm{Ma}$, followed by extensive loss of duplicate genes (32). Considerable sequence blocks shared synteny with the chicken genome, some of which represented 1:1 relationship with chicken microchromosomes. The Australian lungfish genome, though much expanded with repetitive sequence, also possesses many microchromosomes with homology to amphioxus chromosomes (37).

We aligned the bird (emu) and amphioxus genomes (Fig. $3 E$ and SI Appendix, Fig. S3). Of the 21 emu microchromosomes with sufficient shared sequence to detect homology, nine bird microchromosomes each represented a single amphioxus chromosome, suggesting that ancestral chromosomes have been retained intact. Another six emu microchromosomes each contained sequences from two amphioxus chromosomes, implying fusions in ancestral vertebrates.

We then aligned single amphioxus chromosomes to the emu genome, demonstrating that most have strong homology to one or two microchromosomes, as well as to two (sometimes three) regions of macrochromosomes (Fig. $3 F$ and SI Appendix, Fig. S4). These probably represent the four copies of the chordate genome that resulted from two rounds of doubling. It is notable that one or two of these homologies (usually including at least one microchromosome) are strong and focused, whereas the others are more dispersed, suggesting rearrangement, deletion, and repeat expansion. This suggests that all copies of each ancient chromosome have retained synteny (physical linkage), with a single copy equating to one or more microchromosomes, as previously observed (32).

Unsurprisingly, given their homology to bird microchromosomes, most turtle, snake, and lizard microchromosomes also equated to single or fused amphioxus chromosomes. The most parsimonious explanation is that reptile/bird microchromosomes represent ancestral chordate chromosomes (or fused chromosomes) that have retained synteny.

We conclude that reptile and bird microchromosomes are relics of an original animal genome with tiny, gene-rich chromosomes, represented today by amphioxus. Since turtle and squamate microchromosomes are different subsets of bird microchromosomes, this implies that these ancestral microchromosomes have been progressively lost by fusion at different rates independently in different lineages. Fusion has evidently been slower in birds than in other reptile lineages.

Fusion of Microchromosomes into Macrochromosomes. To examine the process by which ancient microchromosomes became incorporated into macrochromosomes in vertebrates, we analyzed the genomes of exceptional reptile and bird species in which fusion has removed many or most microchromosomes. We aligned the genome of emu (representing ancestral birds) with alligator (representing crocodilians that diverged from birds $240 \mathrm{Ma}$ ) and eagle (which shared a common ancestor with other birds with a conserved karyotype only about $80 \mathrm{Ma}$ ).

We found that emu macro- and microchromosome sequence had obvious homologs in the alligator and eagle genomes, although they were considerably rearranged (Fig. $3 G$ ). Strong homology between emu and alligator macrochromosome arms shows that the five very large alligator chromosomes are all fusions and rearrangements of ancestral macrochromosomes, as was demonstrated by chromosome painting between the chicken and the Nile crocodile $(21,38)$, which shares many chromosomes with alligator (28). An alligator macrochromosome and the largest microchromosome were each generated by fusions of an ancestral macro- and microchromosome. However, the 10 smallest alligator chromosomes each comprise either single or fused ancestral microchromosomes; one small alligator chromosome represents a single microchromosome, five alligator chromosomes represent fusions of two, and four alligator chromosomes fusions of three ancestral microchromosomes. These changes must have occurred in the crocodilian lineage in the $\sim 240$ My since they shared a common ancestor with birds. It is striking that all but two rearrangements are either a macromacro or a micromicro fusion.

The rearranged eagle genome, in contrast, contains many fusions between macro- and microchromosomes and many fissions of ancestral macrochromosomes into smaller chromosomes (Fig. 3G). Only three macrochromosomes have been retained intact; two have undergone centric rearrangements of 
chromosome arms and two have each fused with an ancestral microchromosome. The other four ancestral macrochromosomes have undergone multiple fissions, the products of which have fused to other macrochromosome arms or ancestral microchromosomes. The largest emu chromosome has undergone fission into seven regions, six of which are present as small chromosomes (five in the micro range). In addition, two small eagle macrochromosomes derive from micromicro fusions.

Thus, eagle microchromosomes include only four ancestral microchromosomes and one micromicro fusion. Three ancestral microchromosomes have fused to form macrochromosome arms or terminal regions. What is striking is that six eagle microchromosomes were derived from regions (mostly terminal) of ancestral macrochromosomes so represent de novo microchromosomes.

Thus, in both alligator and eagle, macrochromosome arms and microchromosomes have been fused with each other, and with the termini of macrochromosomes. However, the patterns of fusions and fissions are quite different in the eagle and alligator, attesting to independent rearrangement facilitated by different mechanisms.

Microchromosome Interactions. Our cytogenetic studies (discussed above and see Fig. 2) confirm and extend previous observations (4) that microchromosomes are spatially segregated within cells, occupying a central location at interphase, and during mitosis and meiosis, in turtles and squamates as well as birds. We also showed that microchromosomes may retain this central position even after fusion to macrochromosomes, implying that size alone does not determine microchromosome location.

Data from high-throughput chromosome conformation capture (Hi-C) now provide a molecular description of this spatial segregation. Hi-C data incorporated in the new emu assembly (35) and the rattlesnake assembly (39) reveal that microchromosomes interact with each other more than with macrochromosomes in these species, as has been observed also in other birds, snakes, and turtles (40). We confirmed and extended these observations to other birds and reptiles (green sea turtle, alligator, python, eagle, tegu, emu, greater rhea, and water dragon), using DNA Zoo Hi-C data (41) (SI Appendix, Fig. S1 $A-G)$. These species show various degrees of rearrangement of ancestral macro- and microchromosomes, permitting us to determine whether microchromosome fusion or macrochromosome fission can alter the GC ratio and interaction characteristics.

On average, pairs of loci borne on the same chromosome present higher interaction probabilities (intrachromosomal interactions) than between loci on different chromosomes (interchromosomal interactions). At the genome-wide level, this cis/trans interaction pattern reflects chromosomal territoriality (42). As expected, the interaction between neighboring loci on the same chromosome decreases as genomic distance increases. This reduction in interactions can be represented as genomic distance-dependent contact probabilities $[\mathrm{P}(\mathrm{s})]$, representing the level of chromosome compaction (43).

Genome-wide heat-map plots of representative species ( $S I$ Appendix, Fig. S1 $A-G$ ) all show that, as well as a high GC ratio, there is a higher interchromosomal interaction and higher distant dependent contact probabilities $[\mathrm{P}(\mathrm{s})]$ between microchromosomes than between macrochromosomes or between macro- and microchromosomes. More intense interaction is therefore an intrinsic feature of all reptile/bird microchromosomes, reflected by their arrangement within the nucleus during interphase (Fig. 2).

Given that this interaction pattern is an ancestral feature of microchromosomes, we asked whether rearrangements in alligator, water dragon, python and eagle resulted in changes of properties of ancestral and de novo microchromosomes.

The alligator has chromosomes out of the microchromosome size range that were formed by micromicro fusions (Fig. $3 G$ ). We found that these fused microchromosomes retain their higher GC ratio and still interact strongly with each other and the smaller microchromosomes (SI Appendix, Fig. S1).

In the water dragon, several ancestral microchromosomes are fused to form four new regions of macrochromosomes. These are also present in the python genome (SI Appendix, Fig. S1I) so must have occurred in a common squamate ancestor about $180 \mathrm{Ma}$. When we plotted GC content and contact probabilities according to the ancestral state (SI Appendix, Fig. S1F) we found that these ancestral microchromosomes fused to macrochromosomes had a lower GC content but retained their high contact probability. However, two ancestral microchromosomes that were more recently fused to either end of the largest scaffold only in python still retain their elevated GC content (SI Appendix, Fig. S1G). This suggests that the GC-richness of microchromosomes is retained on rearrangement but erodes over time.

The much-rearranged eagle genome, for which there is both chromosome-level assembly and $\mathrm{Hi}-\mathrm{C}$ data, allowed us to assess features of both microchromosomes which fused to form macrochromosomes, and macrochromosomes that were split into microchromosomes, in the $\sim 80$ My since the eagle last shared a common ancestor with condors, which retain an ancestral bird karyotype (44).

Of the 30 ancestral microchromosomes present in other birds, only four remain in the eagle; two others are fused into a larger microchromosome and 12 are fused with macrochromosomes. Nine new microchromosomes represent regions of ancestral macrochromosomes which underwent fission (Fig. $3 G)$.

Unlike other bird species (SI Appendix, Fig. S1), the eagle micro- and macrochromosomes showed no abrupt differences in GC content or contact probabilities with size (Fig. 4), reflecting their recent reshuffling. The microchromosomes still retained elevated interchromosomal interactions (Fig. 4), although this was not as well correlated with chromosome size as for other species.

To explore the origin of these differences, we examined the characteristics of eagle sequence according to ancestral state as deduced from comparison with the emu genome (Fig. 3G). Regions of synteny with the emu genome were classified as macro (macro in both species), micro (micro in both species), new macro (micro in emu but macro in eagle), or new micro (macro in emu but micro in eagle). We found that GC content reflected the ancestral state (Fig. 4). The microchromosomes incorporated into macrochromosomes (new macros) in eagle maintained high GC content, and macrochromosomes broken down to microchromosome size (new micros) retained their low GC content.

In contrast, the distance-dependent contact probabilities $[\mathrm{P}(\mathrm{s})]$ of these four classes of regions did not consistently reflect their ancestral state. As expected, ancestral macrochromosomes displayed lesser and microchromosomes greater distancedependent interaction probabilities (Fig. 4). Microchromosomes incorporated into macrochromosomes also maintained relatively high contact probabilities at genomic distances below $1 \mathrm{Mbp}$, albeit slightly lower than the ancestral microchromosomes. Surprisingly, however, the new microchromosomes derived recently from macrochromosomes had almost the same elevated distance-dependent interaction probabilities as the ancestral microchromosomes. This cannot be a consequence of altered base ratio, since percent GC was not increased, and may reflect different levels of chromatin compaction or compartmentalization. 
We conclude that the high interaction probabilities of ancestral microchromosomes are maintained after their incorporation into a macrochromosome but erode over time. However, novel microchromosomes rapidly adopt high interaction with other microchromosomes.

The Fate of Microchromosomes in Mammals. To explore the fate of ancient microchromosomes in therian mammals (eutherian and marsupial mammals), we compared the genomes of emu with those of koala (a marsupial) and human (eutherian) (Fig. $3 H$ ). We observed regions with some homology to bird microchromosomes, though these were weak and dispersed. There was one region of the human genome (chromosome 17) and two regions of the koala genome that had homology to two or more microchromosomes but no evidence that microchromosomes are retained intact in either species.

Monotremes (egg-laying mammals such as the platypus) have a karyotype somewhat resembling those of reptiles, with 6 pairs of large chromosomes and 20 pairs of much smaller chromosomes (45). Although the small platypus chromosomes are not in the size range of reptile microchromosomes, we asked whether these were the vestiges of ancestral microchromosomes. We found that most platypus chromosomes, large and small, had contributions from multiple chicken macro- and microchromosomes, and there was no obvious enrichment of microchromosomes in the small platypus chromosomes (Fig. $3 H)$.

However, homology with ancestral microchromosomes was not distributed randomly within the platypus genome. Four platypus chromosomes appear to be entirely composed of regions with homology to one to four emu microchromosomes, suggesting that they evolved from multiple microchromosome fusions. Other regions with strong homology lay at the ends or comprised arms of platypus chromosomes.

This suggests that in the reptile-like ancestor of mammals micromicro fusions were common (as they were also in the crocodilian ancestor). These blocks of fused microchromosomes have been preserved in platypus macrochromosomes but were disrupted in therian mammals, so that little vestige remains. The high rate of genome reshuffling in mammals contrasts with the stability of genomes in other amniote lineages.

\section{Discussion}

Our analysis of sequence data confirms that reptile and bird microchromosomes, as well as macrochromosomes, are highly conserved within bird, turtle, and squamate lineages, and even between these lineages. Microchromosomes are most numerous and almost invariant among birds, many species having 31 wellconserved microchromosomes. Different microchromosome subsets have been retained in turtles and squamates, implying that the 31 microchromosomes were present in the common ancestor of birds and reptiles about $300 \mathrm{Ma}$, expanding on the 20 considered by Burt (8) to be ancestral. Previous work using older and more limited sequence datasets implied that chicken microchromosomes represented ancestral birds but suggested that a dinosaur ancestor of birds and crocodiles and an archelosaur ancestor of birds and turtles $>250 \mathrm{Ma}$ had fewer and larger chromosomes that underwent fission (26). Our demonstration that squamates, too, share many microchromosomes with emu implies an older origin.

Strong homology with the small chromosomes of amphioxus implies that the conserved set of bird microchromosomes represent retention of ancient chromosomes of a common ancestor that lived $684 \mathrm{Ma}$. Comparisons of single amphioxus chromosomes with the bird genome reveal homology to four regions, one or two with bird microchromosomes and two or three with bird macrochromosomes. Presumably these four regions of demonstrable homology reflect paralogous sequences generated by the two genome doublings early in vertebrate evolution $(32,46)$. For most of these paralogous regions one or two of the strongest are located on bird microchromosomes, probably representing paralogous sequences that retain the gene richness and low repetitive sequence content of the original chromosomes, whereas other genome copies have suffered deletion, transposable element invasion, and rearrangement. It is interesting to speculate that microchromosomes may be protected from rearrangement and insertion of repetitive elements by their longer subtelomere regions, their spatial isolation, and high interaction.

Microchromosomes as well as macrochromosomes have been proportionately lengthened by insertion of transposable elements, as observed over many vertebrates (47), occasionally moving out of the microchromosome range. However, most microchromosomes have been progressively lost by fusion, and only very occasionally gained by fission of macrochromosomes, in all bird and reptile lineages (Fig. 5).

Loss of microchromosomes in birds and turtles by fusion with macrochromosomes has been documented in several cytological studies (e.g., ref. 48). Patterns of microchromosome loss (Fig. 5) were examined by comparing the genomes of conserved representatives of birds, turtles, and squamates. Almost all loss can be attributed to fusion of two, or sometimes three, microchromosomes to form larger chromosomes, as well as fusion of microchromosomes to macrochromosomes. These fusions are almost always to terminal locations or via centric fusion to constitute a chromosome arm, as was implied by early observations of interstitial and centromeric telomere sequence (49).

Patterns of microchromosome loss were also examined by following the chromosome changes in exceptional species (alligator and golden eagle) with more rearranged chromosomes as shown by cytogenetic and sequencing methods (50). Our extensive analysis showed that these genomes differ from the standard simply by more fusions of the same types that distinguish turtle and squamate genomes, suggesting that the same processes occurred, but at much higher rates.

The onset of instability in a lineage could be quite sudden. For instance, extensive genome remodeling in the eagle lineage must have occurred in the last 80 My since eagles shared a common ancestor with condors, which retain a near-ancestral genome arrangement (44). Cormorants diverged from the same conserved lineage a short time later. Our analysis of the cormorant assembly (51) (Fig. 3D) revealed a rearranged genome; however, few of the rearrangements are shared with eagles. The high rate of independent changes in cormorants or eagles suggests that instability was introduced to the genome of a

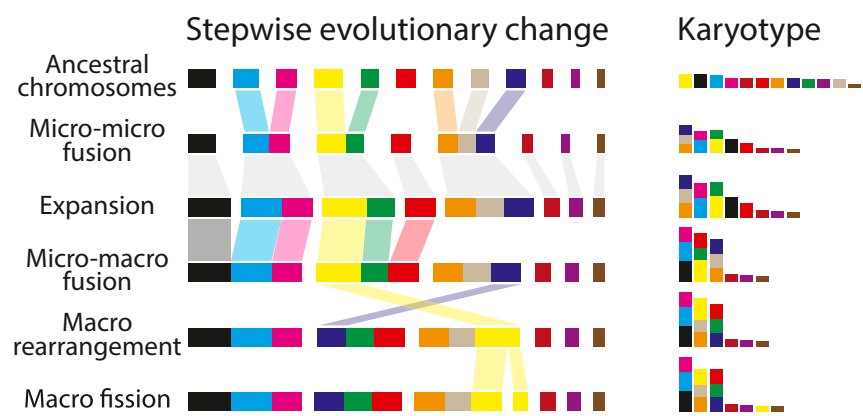

Fig. 5. Evolutionary loss and gain of microchromosomes has reshaped the amniote kartyotype. Fusion between microchromosomes, expansion by the insertion of transposable elements, fusion with macrochromosomes, and macrochromosome rearrangement have led to a reduction of microchromosomes. Rarely macrochromosome fissions result in new microchromosomes. 
common ancestor but was expressed independently in the two lineages. One explanation of the sudden onset of instability after millions of years of extreme conservation is that the genome of an eagle ancestor $(\sim 80 \mathrm{Ma})$ was invaded by a transposable element which facilitated interchromosome rearrangements (52).

Intriguingly, the patterns of microchromosome fusions are quite different in the two exceptional species we examined. Rearrangements in the alligator are almost all confined to micromicro and macromacro fusions, whereas in the eagle they are largely micromacro fusions. Assortative fusions (micromicro and macromacro) may be favored by the spatial compartmentalization $(53,54)$. However, it is likely that rearrangements are driven by the location and type of repetitive elements inserted, as seems to be the case for parrots (27). Transposable elements are ubiquitous in reptiles and birds (55) but are not randomly distributed in the genome (56). For instance, there are several repetitive sequences that are shared only by microchromosomes (57) (e.g., Fig. 2C), which may favor micromicro rearrangements, such as those that predominate in alligator. Other transposable elements are observed to cluster at centromeres of all chromosomes (58) and may be involved in Robertsonian fusions between macro- and microchromosomes.

Loss of microchromosomes has, rarely, been offset in some lineages by the creation of novel microchromosomes from macrochromosome fragments. Several appear in the eagle genome, and there are one or two in other birds (e.g., hummungbird) and squamates (e.g., common lizard).

Reptile and bird microchromosomes are distinguished by their high gene density and high GC content, and particularly by their compartmentalization in the center of interphase and dividing cells. We have documented differential staining (denoting differences in sequence and conformation) and spatial segregation in a variety of squamates and turtles as well as birds consistent with many earlier cytological observations (4). The molecular underpinnings of these properties were revealed by our analysis of chromosome conformation capture data, which shows that microchromosomes in bird, turtle, and squamate lineages all show high interaction within this compartment. These intense interchromosome relationships have been thought to denote different chromatin organization of microchromosomes that reflects some functional coherence.

We analyzed rearranged genomes to examine properties of regions whose status has changed between micro- and macrochromosome. We found that high interaction, as well as high GC content, is retained by ancestral microchromosomes that fused with macrochromosomes in eagle. This is consistent with our cytological observation that two ancestral microchromosomes fused to a macrochromosome in a turtle retain their central position (Fig. $2 E$ ), as was also observed for the microchromosome uniquely fused to chromosome 4 in chickens (10) and fused microchromosomes in the rearranged genomes of falcons and parrots (50). However, high GC content does appear to erode with time, as shown by a lower GC content of fused microchromosomes in snakes.

We discovered, unexpectedly, that novel microchromosomes derived by fission of ancestral macrochromosomes rapidly acquired an increased distance-dependent interaction probability, although their GC content stayed low. This suggests that intense interaction may be the result not of sequence composition but of chromosome compaction or location in the cell, which may be influenced by epigenetic factors or chromosome size.

The extreme conservatism of the bird/reptile genomes, and even the modest rearrangements that characterize the crocodilians, expose the flagrant chromosome shuffling in mammals as a glaring exception among amniotes. Mammals have extraordinarily variable karyotypes. In eutherian mammals, a near- identical-sized $(3 \mathrm{~Gb})$ genome is packaged as anything between 3 pairs of enormous chromosomes in the Indian muntjac to 51 pairs of small chromosomes in the red viscacha rat (59). Not only is the highly rearranged eutherian genome subdivided into a few large or many small chromosomes but sequences have been scrambled in many lineages. Large synteny blocks are shared by some species (e.g., humans, cats, and even sloths), enabling reconstruction of ancestral eutherian karyotypes (60, 61 ), but even these blocks of shared synteny are wildly different in other species, especially the rodents. The marsupial karyotype, in contrast, is highly conserved between all 260 species and derives from a $n=7$ basal karyotype largely by Robertsonian translocations (62).

We found that in eutherians and marsupials microchromosomes have completely disappeared, visible only as broken up patches of homology peppering the genome.

Even the early-branching monotreme mammals, which have a rather reptile-like karyotype with six large and many small chromosomes, retain none of the ancestral microchromosomes. Most of the small platypus chromosomes have homology to several regions of macro- and microchromosomes. However, two large and two small platypus chromosomes seem to be completely made up of fusions of several ancestral microchromosomes, suggesting that the process of amalgamation may have started from many micromicro fusions in a mammalian ancestor.

We propose that microchromosome fusion occurred in the ancestor of all mammals after divergence from the reptile/bird lineage $310 \mathrm{Ma}$ and before the divergence of monotremes from therians $188 \mathrm{Ma}$. These microchromosome blocks must have already undergone major sequence reshuffling before the marsupialeutherian divergence $168 \mathrm{Ma}$ because microchromosome-homologous sequences are split up and distributed all over the genome in both lineages.

The sequence shuffling and size variation among eutherian chromosomes would require some event that loosened the constraints on rearrangement in a mammalian ancestor, probably invasion and amplification of particular retrotransposons, which provide sites for crossing over between nonhomologs (reviewed in ref. 63).

Thus, mammal genomes are spectacularly atypical among amniotes, displaying variation that has been sometimes credited (e.g., ref. 64) with their successful colonization of varied (terrestial and underground, arboreal, aqueous, even aerial) environments. We need to understand what effects these rearrangements between ancestral macro- and microchromosomes had on genome function. The high gene density, atypical base ratio, spatial segregation, and high interaction between microchromosomes suggests a functional coherence of this part of the genome, which survives in the subsets of microchromosomes retained in birds, turtles, and squamates. Gene-dense and active chromosome regions are also located centrally in mammalian cells (65), but these do not equate with ancestral microchromosomes. It is difficult to unscramble cause-andeffect relationships between chromosome size, location, gene density, GC content, and distance-dependent interaction probabilities.

Our overall conclusion is that bird microchromosomes represent remnants of the original building blocks of vertebrate genome. They retain high gene density and low content of repetitive sequence and share conserved features across all reptile and bird clades. Their progressive fusion with each other, and with macrochromosomes, occurred conservatively and gradually in most reptile lineages, but more rapidly in a few clades. Multiple microchromosome fusions occurred early in mammal evolution and were followed by lineage-specific rearrangement and a huge variety of fusions and fissions that disrupted the relationship between microchromosomes. Among amniotes, even vertebrates, mammal genomes are the true exceptions. 


\section{Materials and Methods}

Cytology. Mitotic and meiotic chromosomes preparations and chromosome paint preparation and painting were performed following protocol described in ref. 63. Repeat mapping was performed following protocol described in ref. 64. Briefly, $200 \mathrm{ng} 5^{\prime}$-Cy3-labeled (AAGG) 8 probe was mixed with $15 \mu \mathrm{L}$ hybridization buffer [ $50 \%$ formamide, $10 \%$ dextran sulfate, $2 \times$ saline sodium citrate (SSC), and $40 \mathrm{mmol} / \mathrm{L}$ sodium phosphate, $\mathrm{pH} 7.0$, and $1 \times$ Denhardt's solution] added to slides containing fixed chromosome preparation and denatured at $68^{\circ} \mathrm{C}$ for $5 \mathrm{~min}$. After denaturation, slides were incubated overnight in a moist hybridization chamber at $37^{\circ} \mathrm{C}$. Slides were then washed once at $60^{\circ} \mathrm{C}$ in $0.4 \times$ SSC and $0.3 \%$ Igepal for $2 \mathrm{~min}$, followed by another wash at room temperature in $2 \times$ SSC and $0.1 \%$ Igepal for $1 \mathrm{~min}$ and air-dried. Slides were then counterstained with DAPI with Vectashield. Image analysis was performed using a Zeiss Axioplan epifluorescence microscope equipped with a charge-coupled device camera (RT-Spot; Zeiss).

Whole-Genome Alignments. One-way all-versus-all LastZ (Release 1.02) (31) alignments were performed for 23 reptile (including birds) species and platypus. Human, Tasmanian devil, and amphioxus genomes were aligned against selected genomes. Alignments were chained and netted using the UCSC Toolkit (http://hgdownload.soe.ucsc.edu/admin/exe/linux.x86_64). Workflow was automated using scripts available at https://github.com/kango2/tiny.

Briefly, the LastZ alignment parameters were $\mathrm{K}=2400 \mathrm{~L}=3000 \mathrm{Y}=9400 \mathrm{H}$ $=2000$-ambiguous = iupac. Chaining was performed with axtChain using -minScore $=3000$-linearGap $=$ medium as parameters. Chains were sorted with chainSort, prenetting was performed with chainPreNet, and netting was performed with chainNet, each using default parameters. Syntenic blocks were calculated with netSyntenic.

Homology and statistics were plotted in R using the tidyverse package (v1.3.0) with custom scripts available at the GitHub repository. Microchromosome and macrochromosome labels, when not available, were assigned to assembled sequences based on published karyotype data summarized in SI Appendix, Tables S1 and S2.

GC Content and Gene Density. GC content of each scaffold was calculated using BBMap (v38. 9) (65) for DNAzoo data or obtained from the relevant $\mathrm{NCBI}$ genome information website. Gene density per megabase was calculated by dividing the number of annotated genes on a chromosome by its length.

Interscaffold Interactions. The $\mathrm{Hi}-\mathrm{C}$ data were obtained from DNAzoo ( $\mathrm{SI}$ Appendix, Table S1). Hi-C matrices were exported to 50-kb GInteractions bins with HiCExplorer (v3.6) (66). This format consisted of seven columns: origin scaffold, origin scaffold start, origin scaffold end, target scaffold, target scaffold start, target scaffold end, and number of interactions between bins. The largest scaffolds were extracted, equal to the expected number of

1. J. M. Van Brink, L'expression morphologique de la digamétie chez les Sauropsidés et les Monotrèmes. Chromosoma 10, 1-72 (1959).

2. L. Christidis, Animal Cytogenetics 4: Chordata 3 B, Aves (Gebrüder Borntraeger, Berlin, 1990).

3. N. Takagi, M. Sasaki, A phylogenetic study of bird karyotypes. Chromosoma 46, 91-120 (1974).

4. F. A. Habermann et al., Arrangements of macro- and microchromosomes in chicken cells. Chromosome Res. 9, 569-584 (2001).

5. E. H. Newcomer, Accessory chromosomes in the domestic fowl. Genetics 40, 587-588 (1955).

6. H. A. McQueen, G. Siriaco, A. P. Bird, Chicken microchromosomes are hyperacetylated, early replicating, and gene rich. Genome Res. 8, 621-630 (1998).

7. J. Smith et al., Differences in gene density on chicken macrochromosomes and microchromosomes. Anim. Genet. 31, 96-103 (2000).

8. D. W. Burt, Origin and evolution of avian microchromosomes. Cytogenet. Genome Res. 96, 97-112 (2002)

9. I. Nanda, M. Schmid, Localization of the telomeric (TTAGGG)n sequence in chicken (Gallus domesticus) chromosomes. Cytogenet. Cell Genet. 65, 190-193 (1994).

10. International Chicken Genome Sequencing Consortium, Sequence and comparative analysis of the chicken genome provide unique perspectives on vertebrate evolution. Nature 432, 695-716 (2004).

11. T. M. Degrandi et al., Introducing the bird chromosome database: An overview of cytogenetic studies in birds. Cytogenet. Genome Res. 160, 199-205 (2020).

12. S. Shetty, D. K. Griffin, J. A. Graves, Comparative painting reveals strong chromosome homology over 80 million years of bird evolution. Chromosome Res. 7, 289-295 (1999).

13. D. K. Griffin, L. B. Robertson, H. G. Tempest, B. M. Skinner, The evolution of the avian genome as revealed by comparative molecular cytogenetics. Cytogenet. Genome Res. 117, 64-77 (2007). chromosomes based on the karyotype information (SI Appendix, Table S1). Each square of the matrix represents the mean of normalized interaction values between the extracted scaffolds at $50-\mathrm{kb}$ bin resolution.

Distance-Dependent Contact Probability P(s). The Hi-C matrices were exported with HiCExplorer (v3.6) to h5 format. The scaffolds for each species were classified as either macro- or microchromosomes (SI Appendix, Table S2). Using the hicAdjustMatrix function, independent matrices were created for macrochromosomes and microchromosomes. Distance-dependent contact probabilities $[P(s)]$ were calculated using hicPlotDistVsCounts (from the HiCExplorer package), which were plotted with a maximum distance of $1 \times 10^{8} \mathrm{bp}$.

Golden Eagle Analysis. Regions of the golden eagle genome were classified according to homology with the chicken genome. These were macro (macro in both species), micro (micro in both species), new macro (micro in chicken but macro in eagle), or new micro (macro in chicken but micro in eagle). These newly created scaffolds were then used to calculate GC content and distancedependent contact probabilities as described above.

Data Availability. Genome data used in the publication are all publicly available (see SI Appendix, Table S1). All coordinates of pairwise alignment chains are available for download at https://cloudstor.aarnet.edu.au/plus/s/ twyXXROkfpRZJiu. Due to large file sizes ( 3 TB total), raw alignments can be provided upon request. All code and metadata used for automating wholegenome alignments and plotting of figures are available at https://github. com/kango2/tiny

ACKNOWLEDGMENTS. P.K. gratefully acknowledges the resources provided by University of Western Australia, the Aiden laboratory at Baylor College of Medicine, DNA Zoo consortium partners, and additional computational resources and support from the Pawsey Supercomputing Centre with funding from the Australian Government and the Government of Western Australia. Unpublished genome assemblies and sequencing data for tegu, eastern water dragon, burmese python, greater rhea, band-tailed pigeon, golden eagle, American alligator, green sea turtle, koala, and emu are used with permission from the DNA Zoo Consortium (https://www.dnazoo.org/). P.D.W., A.G., J.A. M.G., F.G., T.E., and C.F. are supported by grants from the Australian Research Council (DP170101147, DP180100931, DP210103512, and FT200100192). H.R.P. is supported by an Australian National University research fellowship. This work was supported by computational resources provided by the Australian Government through National Computational Infrastructure under the National Computational Merit Allocation Scheme and Australian National University Merit Allocation Scheme. A.R.-H. is supported by the Spanish Ministry of Economy and Competitiveness (CGL2017-83802-P) and by the Spanish Ministry of Science and Innovation (PID2020-112557GB-I00). L.A.-G. is supported by an FPI predoctoral fellowship from the Spanish Ministry of Economy and Competitiveness (PRE-2018-083257).

14. R. Kretschmer, M. A. Ferguson-Smith, E. H. C. de Oliveira, Karyotype evolution in birds: From conventional staining to chromosome painting. Genes (Basel) 9, 181 (2018).

15. L. G. Kiazim et al., Comparative mapping of the macrochromosomes of eight avian species provides further insight into their phylogenetic relationships and avian karyotype evolution. Cells 10, 362 (2021).

16. R. E. O'Connor et al., Patterns of microchromosome organization remain highly conserved throughout avian evolution. Chromosoma 128, 21-29 (2019).

17. R. Kretschmer et al., Interspecies chromosome mapping in caprimulgiformes, piciformes, suliformes, and trogoniformes (aves): Cytogenomic insight into microchromosome organization and karyotype evolution in birds. Cells 10, 826 (2021).

18. J. Damas, J. Kim, M. Farré, D. K. Griffin, D. M. Larkin, Reconstruction of avian ancestral karyotypes reveals differences in the evolutionary history of macro- and microchromosomes. Genome Biol. 19, 155 (2018).

19. G. Zhang et al., Comparative genomics reveals insights into avian genome evolution and adaptation. Science 346, 1311-1320 (2014).

20. E. Olmo, Trends in the evolution of reptilian chromosomes. Integr. Comp. Biol. $\mathbf{4 8}$, 486-493 (2008)

21. J. E. Deakin, T. Ezaz, Understanding the evolution of reptile chromosomes through applications of combined cytogenetics and genomics approaches. Cytogenet. Genome Res. 157, 7-20 (2019).

22. S. M. I. Alam, S. D. Sarre, D. Gleeson, A. Georges, T. Ezaz, Did lizards follow unique pathways in sex chromosome evolution? Genes (Basel) 9, 239 (2018).

23. Y. Matsuda et al., Highly conserved linkage homology between birds and turtles: Bird and turtle chromosomes are precise counterparts of each other. Chromosome Res. 13, 601-615 (2005).

24. W. Becak, M. L. Becak, H. R. Nazareth, S. Ohno, Close karyological kinship between the reptilian suborder serpentes and the class aves. Chromosoma 15, 606-617 (1964). 
25. Y. Uno et al., Inference of the protokaryotypes of amniotes and tetrapods and the evolutionary processes of microchromosomes from comparative gene mapping. PLoS One 7, e53027 (2012).

26. R. E. O'Connor et al., Reconstruction of the diapsid ancestral genome permits chromosome evolution tracing in avian and non-avian dinosaurs. Nat. Commun. 9, 1883 (2018).

27. Z. Huang et al., Recurrent chromosome reshuffling and the evolution of neo-sex chromosomes in parrots. bioRxiv [Preprint] (2021). http://doi.org/10.1101/2021.03.08 434498 (Accessed 22 June 2021).

28. V. C. S. Oliveira et al., Revisiting the karyotypes of alligators and caimans (Crocodylia, Alligatoridae) after a half-century delay: Bridging the gap in the chromosomal evolution of reptiles. Cells 10, 1397 (2021).

29. S. R. Voss et al., Origin of amphibian and avian chromosomes by fission, fusion, and retention of ancestral chromosomes. Genome Res. 21, 1306-1312 (2011).

30. A. Kapusta, A. Suh, Evolution of bird genomes-a transposon's-eye view. Ann. N. Y Acad. Sci. 1389, 164-185 (2017).

31. Y. Nakatani, H. Takeda, Y. Kohara, S. Morishita, Reconstruction of the vertebrate ancestral genome reveals dynamic genome reorganization in early vertebrates. Genome Res. 17, 1254-1265 (2007).

32. O. Simakov et al., Deeply conserved synteny resolves early events in vertebrate evolution. Nat. Ecol. Evol. 4, 820-830 (2020).

33. R. S. Harris, "Improved pairwise alignment of genomic DNA," PhD thesis, The Penn sylvania State University, State College, PA (2007).

34. M. Kohn, H. Kehrer-Sawatzki, W. Vogel, J. A. M. Graves, H. Hameister, Wide genome comparisons reveal the origins of the human $\mathrm{X}$ chromosome. Trends Genet. 20, 598-603 (2004).

35. J. Liu et al., A new emu genome illuminates the evolution of genome configuration and nuclear architecture of avian chromosomes. Genome Res. 31, 497-511 (2021).

36. R. A. Pyron, F. T. Burbrink, J. J. Wiens, A phylogeny and revised classification of Squamata, including 4161 species of lizards and snakes. BMC Evol. Biol. 13, 93 (2013).

37. A. Meyer et al., Giant lungfish genome elucidates the conquest of land by vertebrates. Nature 590, 284-289 (2021).

38. F. Kasai, P. C. O'Brien, M. A. Ferguson-Smith, Reassessment of genome size in turtle and crocodile based on chromosome measurement by flow karyotyping: Close similarity to chicken. Biol. Lett. 8, 631-635 (2012).

39. D. R. Schield et al., The origins and evolution of chromosomes, dosage compensation, and mechanisms underlying venom regulation in snakes. Genome Res. 29, 590-601 (2019).

40. B. W. Perry, D. R. Schield, R. H. Adams, T. A. Castoe, Microchromosomes exhibit distinct features of vertebrate chromosome structure and function with underappreciated ramifications for genome evolution. Mol. Biol. Evol. 38, 904-910 (2021).

41. O. Dudchenko et al., De novo assembly of the Aedes aegypti genome using $\mathrm{Hi}-\mathrm{C}$ yields chromosome-length scaffolds. Science 356, 92-95 (2017).

42. B. R. Lajoie, J. Dekker, N. Kaplan, The Hitchhiker's guide to Hi-C analysis: Practical guidelines. Methods 72, 65-75 (2015).

43. J. Dekker, M. A. Marti-Renom, L. A. Mirny, Exploring the three-dimensional organization of genomes: Interpreting chromatin interaction data. Nat. Rev. Genet. 14, 390-403 (2013).
44. T. Raudsepp et al., Cytogenetic analysis of California condor (Gymnogyps californianus) chromosomes: Comparison with chicken (Gallus gallus) macrochromosomes. Cytogenet. Genome Res. 98, 54-60 (2002).

45. D. McMillan et al., Characterizing the chromosomes of the platypus (Ornithorhynchus anatinus). Chromosome Res. 15, 961-974 (2007)

46. T. D. Lamb, Analysis of paralogons, origin of the vertebrate karyotype, and ancient chromosomes retained in extant species. Genome Biol. Evol. 13, evab044 (2021).

47. A. Canapa, M. Barucca, M. A. Biscotti, M. Forconi, E. Olmo, Transposons, genome size, and evolutionary insights in animals. Cytogenet. Genome Res. 147, 217-239 (2015).

48. P. E. Lithgow et al., Novel tools for characterising inter and intra chromosomal rearrangements in avian microchromosomes. Chromosome Res. 22, 85-97 (2014).

49. I. Nanda et al., Distribution of telomeric (TTAGGG)(n) sequences in avian chromosomes. Chromosoma 111, 215-227 (2002).

50. R. E. O'Connor et al., Chromosome-level assembly reveals extensive rearrangement in saker falcon and budgerigar, but not ostrich, genomes. Genome Biol. 19, 171 (2018).

51. A. Burga et al., A genetic signature of the evolution of loss of flight in the Galapagos cormorant. Science 356, eaal3345 (2017).

52. J. Zhang, C. Yu, L. Krishnaswamy, T. Peterson, Transposable elements as catalysts for chromosome rearrangements. Methods Mol. Biol. 701, 315-326 (2011).

53. T. Marques-Bonet, A. Navarro, Chromosomal rearrangements are associated with higher rates of molecular evolution in mammals. Gene 353, 147-154 (2005).

54. Y. Zhang et al., Spatial organization of the mouse genome and its role in recurrent chromosomal translocations. Cell 148, 908-921 (2012).

55. D. Kordis, Transposable elements in reptilian and avian (sauropsida) genomes. Cytogenet. Genome Res. 127, 94-111 (2009).

56. T. Sultana, A. Zamborlini, G. Cristofari, P. Lesage, Integration site selection by retroviruses and transposable elements in eukaryotes. Nat. Rev. Genet. 18, 292-308 (2017)

57. F. Grützner et al., Chicken microchromosomes are hypermethylated and can be identified by specific painting probes. Cytogenet. Cell Genet. 93, 265-269 (2001).

58. G. Hartley, R. J. O'Neill, Centromere repeats: Hidden gems of the genome. Genes (Basel) 10, 223 (2019)

59. A. S. Graphodatsky, P. L. Perelman, S. J. O'Brien, Eds., Atlas of Mammalian Chromosomes (Wiley \& Sons, ed. 2, 2020).

60. J. Kim et al., Reconstruction and evolutionary history of eutherian chromosomes. Proc. Natl. Acad. Sci. U.S.A. 114, E5379-E5388 (2017).

61. M. Svartman, G. Stone, R. Stanyon, The ancestral eutherian karyotype is present in Xenarthra. PLoS Genet. 2, e109 (2006).

62. J. E. Deakin et al., Reconstruction of the ancestral marsupial karyotype from comparative gene maps. BMC Evol. Biol. 13, 258 (2013).

63. S. J. Klein, R. J. O'Neill, Transposable elements: Genome innovation, chromosome diversity, and centromere conflict. Chromosome Res. 26, 5-23 (2018).

64. M. J. D. White, Modes of Speciation (W. H. Freeman, San Francisco, 1978).

65. T. Cremer, C. Cremer, Chromosome territories, nuclear architecture and gene regulation in mammalian cells. Nat. Rev. Genet. 2, 292-301 (2001).

66. T. Ezaz et al., The dragon lizard Pogona vitticeps has ZZ/ZW micro-sex chromosomes Chromosome Res. 13, 763-776 (2005). 